\title{
New HI Features of the Magellanic System
}

\author{
M. E. Putman \\ Mount Stromlo and Siding Spring Observatories, ANU, ACT 2611, \\ Australia \\ B. K. Gibson \\ CASA, University of Colorado, Boulder, CO 80309, USA \\ L. Staveley-Smith \\ Australia Telescope National Facility, CSIRO, NSW 2121, Australia
}

\begin{abstract}
.
The first results from the HI Parkes All-Sky Survey (HIPASS) provide a spectacular view of the global $\mathrm{HI}$ distribution in the vicinity of the Magellanic Clouds and the southern Milky Way. A 2400 square degree mosaic around the South Celestial Pole (SCP) reveals the existence of a narrow, continuous counter-stream which "leads" the direction of motion of the Clouds, i.e., opposite in direction to the Stream. This strongly supports the gravitational model for the Stream in which the leading and trailing streams are tidally torn from the body of the Magellanic Clouds. The data also reveal additional tidal features in the Bridge region which appear to emanate from the LMC, and a distinct spiral structure within the LMC itself.
\end{abstract}

\section{Introduction and Observations}

The gas distribution in the Magellanic Clouds, unlike the stellar component, immediately suggests that the Clouds are not independent entities, but part of a dynamically interacting system. Recent observations taken as part of the HI Parkes All-Sky Survey (HIPASS) reveal several exciting new features of this system. The single dish $21-\mathrm{cm}$ line observations allow us to examine the large scale structure of the LMC and the overall spatial and velocity distribution of the SMC and Bridge gas, and resolve the long-standing controversy about the mechanism responsible for the formation of the Magellanic Stream.

HIPASS is a survey for extragalactic neutral hydrogen in the southern sky $\left(\delta \leq+2^{\circ}\right)$, which has also proven useful for studies of the local gas distribution (i.e., -400 to $+400 \mathrm{~km} \mathrm{~s}^{-1}$ ). The survey is carried out by actively scanning the 64-m Parkes telescope, equipped with the 13 feed-horn multibeam receiver, across the sky in declination (Staveley-Smith 1997). The entire southern sky has already been scanned once with a spacing on the sky of $\sim 7^{\prime}$ and a sensitivity of $20 \mathrm{mK}(1 \sigma)$. The complete survey will consist of five separate scans, resulting 


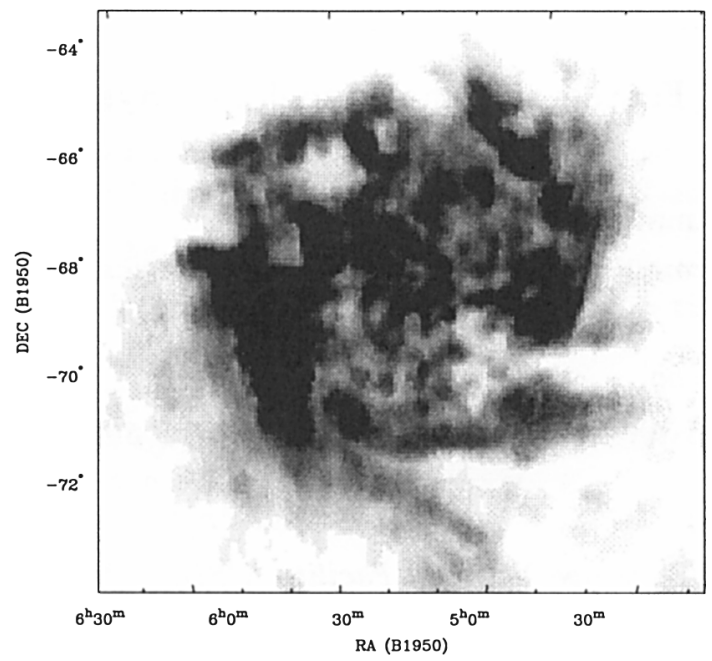

Figure 1. HIPASS map of the LMC. Note the distinct spiral arm in the south and the two stubs in the north.

in sub-Nyquist sampling and a resulting sensitivity of $9 \mathrm{mK}(1 \sigma)$. The channel spacing is $13.2 \mathrm{~km} \mathrm{~s}^{-1}$; after hanning smoothing, the final resolution is 26.4 $\mathrm{km} \mathrm{s}^{-1}$. The survey's unbiased coverage of the southern sky and dense spatial sampling provide us with the ideal tools to reveal previously unidentified HI structure about the Magellanic Clouds.

\section{Results}

The data presented here are from the first scan of HIPASS data. Starting with the LMC (Figure 1), we immediately see the advantages of fully sampled single dish observations. Figure 1 highlights the distinct spiral structure of the LMC and also depicts the diffuse HI edges on all sides of the Cloud. This contradicts the suggestion that the gas is being compressed as the LMC moves through the halo of the Milky Way (e.g., de Boer et al. 1998); however, there is still a steep gradient in the east and the diffuse nature of the outer gas may be due to differential rotation.

The LMC appears to have 3 spiral "arms" (Figure 1) and one southern extension which looks like another thin spiral arm until its relationship to the Magellanic Bridge gas is examined. Figure 2 shows the LMC+Bridge+SMC as seen by HIPASS. In this picture the LMC's southern extension is clearly seen as a tidal feature which merges with gas from the SMC, forming the Bridge. This figure also shows the beginning of the Magellanic Stream which continues for another $100^{\circ}$ to the north (Mathewson et al. 1974). The clumpy nature of the Stream is immediately apparent, even in this limited region.

In addition to the Magellanic Stream, Figure 2 also shows the beginning of a thin southern feature emanating from the SMC+Bridge region $(\ell, b=$ $292^{\circ},-32^{\circ}$ ). Moving out to a 2400 square degree mosaic about the South Celes- 


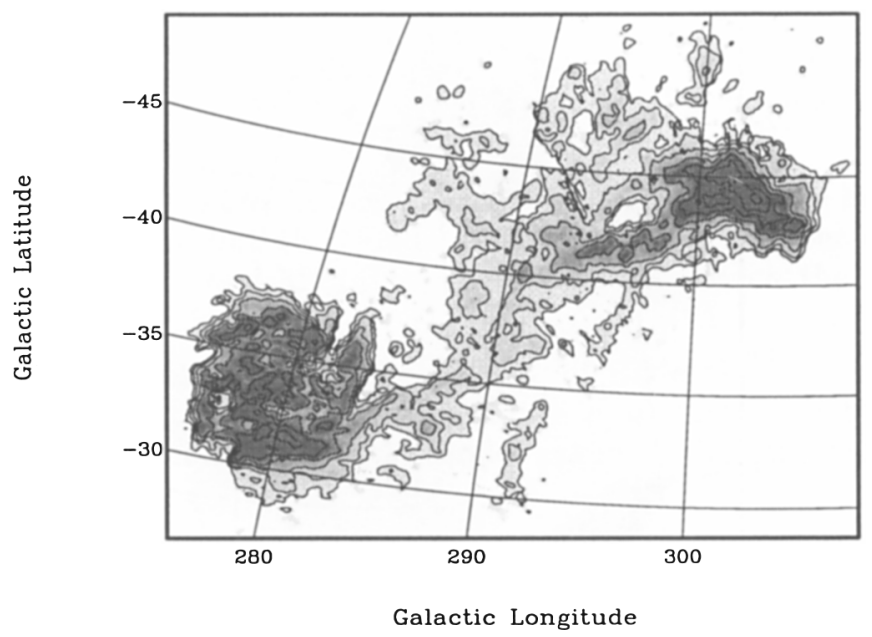

Figure 2. Brightness temperature map of the LMC+SMC+Bridge region including $V_{L S R}=85-400 \mathrm{~km} \mathrm{~s}^{-1}$. Contours are a geometric progression from $1.3-83 \mathrm{~K}$.

tial Pole (Figure 3), we see that this feature continues "towards" the Galactic Plane, on the leading side of the Clouds. This extension is at very high positive velocities and is clearly separate from Galactic emission (see Putman et al. 1998). The leading feature continues from the Clouds both spatially and in velocity, to $b \approx-8^{\circ}$ and $\mathrm{V}_{L S R}=350 \mathrm{~km} \mathrm{~s}^{-1}$.

This leading extension is exactly the type of feature predicted to form during a tidal interaction between the Clouds and the Milky Way (e.g., Gardiner \& Noguchi 1996). There are two principal interaction scenarios which attempt to explain the presence of the Magellanic Stream. One uses gravitational tides to pull out leading and trailing streams from the Clouds; the other employs rampressure stripping to rip the Stream out of the Clouds as they pass through an extended Galactic halo. The argument against tidal models (and for the ram-pressure models) is largely based on two observational features: no leading stream has been detected, and there are no stars observed in the Stream (stars should also be affected by tidal forces). The leading feature of Figure 3 removes the first discriminant against tidal models. The second issue, not finding stars in the Stream, is actually quite complex. It is common to find systems with strong HI tidal features and no optical counterpart (e.g., the M81 system - Yun et al. 1994) or HI streams offset from the optical streams (e.g., Smith et al. 1997). It may be that we have not looked in the right place for this optical counterpart to the Magellanic Stream, or, considering that the Stream is believed to be $\sim 1.5 \mathrm{Gyr}$ old, we may not be looking for the right age of stars (Majewski et al. 1999). In addition, it is probable that the gas in the Clouds was originally more extended than the stars. Thus, the discovery of the Leading Arm is the strongest support in 25 years for a past tidal interaction between the Magellanic Clouds and the Milky Way. 


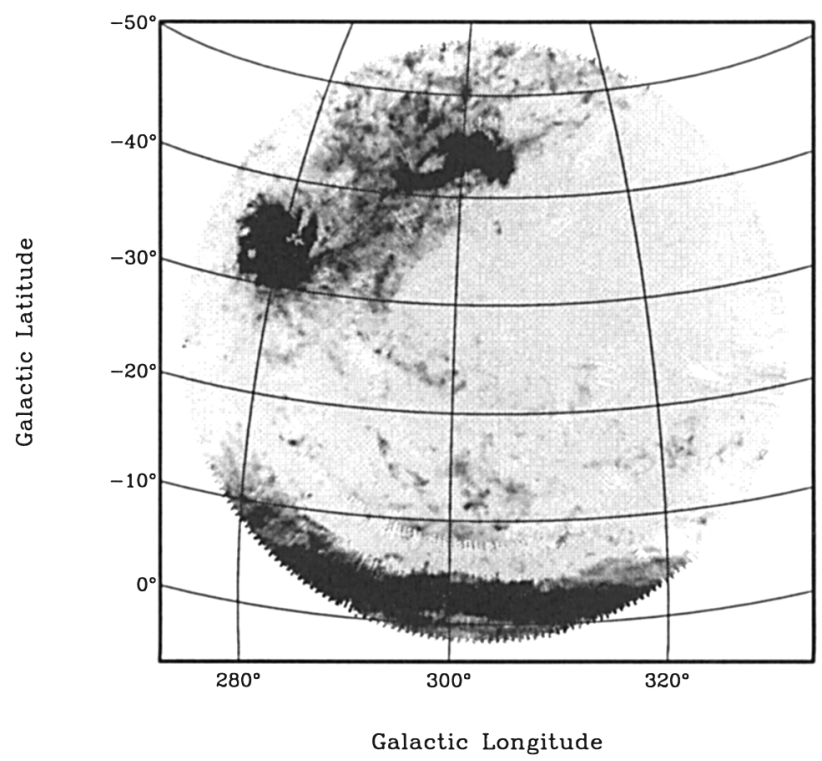

Figure 3. Brightness temperature map of the South Celestial Pole including $V_{L S R}=85-400 \mathrm{~km} \mathrm{~s}^{-1}$.

There are several ways in which the tidal interaction scenario can be confirmed. HIPASS will continue to map the HI about the Magellanic System, allowing us to search for a continuation of the Leading Arm and other Magellanicrelated features. A preliminary look at the data on the other side of the Galactic Plane suggests that the arm veers to the east and continues to $b \approx 30^{\circ}$. We will also increase the sensitivity of our present data by adding more multibeam scans and increase our velocity resolution by observing these features with the narrowband facility. Emission line observations and metallicity determinations will also prove useful for constraining the dominant ionization mechanism in the Leading Arm region and confirming the Magellanic origin.

Acknowledgments. We thank the multibeam working group for their help in the data collection and development of the instrumentation and software.

\section{References}

de Boer, K.S., Braun, J.M., Vallenari, A., \& Mebold, U. 1998, A\&A, 329, L49

Gardiner, L.T., \& Noguchi M. 1996, MNRAS, 278, 191

Majewski, S., et al. 1999, this volume

Mathewson, D.S., Cleary, M.N., \& Murray, J.D. 1974, ApJ, 190, 291

Putman, M.E., Gibson, B.K., Staveley-Smith, L., et al. 1998, Nature, 394, 752

Smith, B.J., Struck, C., \& Pogge, R.W. 1997, 483, 754

Staveley-Smith, L. 1997, PASA, 14, 111

Yun, M.S., Ho, P.T.P., \& Lo, K.Y. 1994, Nature, 372, 530 


\section{Discussion}

Jim Hesser: Are any surveys underway to identify stellar populations associated with your "leading arm"?

Putman: Not presently, but Majewski et al. (this volume) have begun a search for stellar streamers about the clouds and has found a number of giant stars offset from the HI in the LMC. They have plans to search the Leading Arm region as well.

Lance Gardiner: Looking at the global view of $\mathrm{HI}$ in the Magellanic System, would you say that the Leading Arm feature originates from the inter-cloud region? The Gardiner and Noguchi model suggests that the feature emerges nearer the LMC.

Putman: It is difficult to say exactly. There appears to be a filament extending down from the SMC/Inter-Cloud regions into the arm, as well as a more tenuous filament emerging formt he bottom of the LMC. Higher velocity resolution observations should help to clarify this.

Klaas de Boer: Can you tell us what the column densities are in your leading arm? On the other side of the galaxy there are a lot of high velocity clouds belonging to the Milky Way halo. Can you exclude that the clouds you see in front of the LMC are plain normal galactic HVC's?

Putman: The maximum column density is approximately $6 \times 10^{19}$ atoms $\mathrm{cm}^{-2}$ and there does not seem to be a systematic variation in column density along the arm. We do not yet know if high velocity clouds belong to the Milky Way halo. The Leading Arm is distinct from Milky Way gas in several ways. Its velocity $\left(\sim 310 \mathrm{~km} \mathrm{~s}^{-1}\right)$ is much higher than the velocity of the MW disk gas in this direction $\left(\leq 120 \mathrm{~km} \mathrm{~s}^{-1}\right)$. As seen in the channel maps, the arm is connected to the Magellanic Clouds and not to any Galactic emission. It also has a unique continuous structure for over $25^{\circ}$. 